\title{
Théâtre et société en République de Moldavie après l'indépendance
}

Exemple de la pièce Le septième kafana (2001)

\section{Philippe Loubière}

\section{(2) OpenEdition}

\section{Journals}

Édition électronique

URL : https://journals.openedition.org/cher/10491

DOI : 10.4000/cher.10491

ISSN : 2803-5992

\section{Éditeur}

Presses universitaires de Strasbourg

\section{Édition imprimée}

Date de publication : 1 décembre 2013

Pagination : 217-222

ISBN : 978-2-86820-560-5

ISSN : 1968-035X

\section{Référence électronique}

Philippe Loubière, «Théâtre et société en République de Moldavie après l'indépendance », reCHERches [En ligne], 11 | 2013, mis en ligne le 08 février 2022, consulté le 09 février 2022. URL : http:// journals.openedition.org/cher/10491 ; DOI : https://doi.org/10.4000/cher.10491

\section{(c) () (ㅇ)}

Ce(tte) œuvre est mise à disposition selon les termes de la Licence Creative Commons Attribution -

Pas d'Utilisation Commerciale - Partage dans les Mêmes Conditions 4.0 International. 


\title{
Théâtre et société en République de Moldavie après l'indépendance \\ Exemple de la pièce Le septième kafana (2001)
}

\author{
PHILIPPE LOUBIĖRE \\ Université de Paris 3 \\ Traducteur littéraire
}

Bref panorama de la situation du théâtre en Moldavie (Bessarabie) après 1991

Les auteurs dramatiques de Moldavie ont, comme partout dans l'ancienne Union soviétique, accompagné la rupture avec le régime déchu de leurs écrits, pièces de théâtre et scénarios de film, voire de leur présence à la radio ou à la télévision.

Nous en présentons brièvement quatre parmi les plus représentatifs: Nicolae Negru, Dumitru Crudu, Nicoleta Esinencu et Mihai Fusu.

Le plus ancien, Nicolae Negru, est né en 1948. Après des études d'économie, il se fait connaître par des récits et des esquisses humoristiques, puis par des pièces dont certaines ont gagné des prix de dramaturgie organisés par le ministère de la Culture de Moldavie, comme Revine Marea Sarmațiană et Ne întoarcem în Carpați (toutes deux créées en 1998 au théâtre A.-Mateievici et publiées aux éditions Arc, Chişinău), ou encore Realitatea violetă (s.d.).

Dumitru Crudu est né en 1968; il fait ses études à Chişinău et à Tiflis (Géorgie), puis à Braşov et à Sibiu (Roumanie). Journaliste culturel, il écrit de la poésie et de la prose et participe, en 1999, au lancement d'un nouveau manifeste littéraire, le «Fracturisme». 
Ses écrits sont publiés tant en Moldavie qu'en Roumanie. Dans ce dernier pays, il gagne, en 2003, le prix de la meilleure pièce de l'année, dans un concours organisé par l'Union des Théâtres de Roumanie (Uniter), pour Alegerea lui Alexandru Sutto ("L'élection de Alexandre Sutto»). Il écrit également Steaua fără... Mihail Sebastian (L'étoile sans... Mihail Sebastian) (Éd. Cartea Românescă, 2006) et participe à la mise en écriture d'A şaptea Kafana (Le septième kafana, 2001).

Nicoleta Esinencu, née en 1978, est la fille de Nicolae Esinencu, scénariste de films renommé en Moldavie et en Roumanie. Elle étudie l'art dramatique à l'université d'État de Chişinău, et se fait remarquer par des pièces à la vulgarité un peu forcée, comme Fără zahăr (Sans sucre), qui malmène le personnage de son propre père, ou Fuck you, Eu. Ro. Pa!

Elle aussi participe à la mise en écriture d'A şaptea Kafana.

Mihai Fusu, né en 1960, est le fils du grand acteur de théâtre, metteur en scène et réalisateur moldave Dumitru Fusu, fondateur en 1968 du Teatrul unui Actor ( «Le théâtre d'un Acteur»). Mihai Fusu est, lui aussi, comédien et metteur en scène, d'abord au théâtre Eugène-Ionesco de Chişinău; par la suite, en 2006, il prend la suite de son père à la direction du Teatrul unui Actor. Une tournée internationale entre 1992 et 1994, avec Cântăreața chială, Asteptându-l pe Godot et Regele moare (respectivement La cantatrice chauve, En attendant Godot et Le roi se meurt), jouées en roumain, fait connaître Mihai Fusu en dehors de la Moldavie et de l'ancienne URSS, en France puis en Suisse en particulier.

Ses spectacles les plus notables sont: RASSM (pour «République Autonome Soviétique Socialiste Moldave»), montage musical de poèmes à tonalité avant-gardiste écrits en langue roumaine dans les années 1920, dans ladite RASSM, c'est-à-dire la première Transnistrie aujourd'hui ukrainienne, créée par Staline. Ce montage créé en 1993, a été joué en France en 2011. Mihai Fusu monte ensuite, en 1994, Noi «Nous» un spectacle sur la guerre civile de 1992. Il est également coauteur de Cutia neagră a Moldovei, («La boîte noire de Moldavie») film sur les événements du 7 avril 2010 à Chişinău. Il est aussi et surtout la cheville ouvrière d'A şaptea Kafana, en 2001, dont il est le coauteur avec Dumitru Crudu et Nicoleta Esinencu et le metteur en scène.

Depuis l'alternance politique de 2011, Mihai Fusu est présentateur et réalisateur de l'émission RePublika, chaque dimanche soir, sur la chaîne de télévision moldave PublikaTV. 


\section{Du théâtre documentaire au théâtre engagé dans la prophylaxie sociale}

On voit, notamment dans le travail d'acteur et de metteur en scène de Mihai Fusu, la très grande part que les faits de société ont prise dans l'inspiration dramatique. C'est ce qui a poussé les trois derniers dramaturges cités plus haut à rendre compte d'un phénomène grave qu'a connu la Moldavie, dès les premières années de l'indépendance, acquise en 1991, à savoir la prostitution forcée à l'étranger de jeunes femmes désireuses d'échapper à la pauvreté et à la monotonie de la vie post-soviétique et inconscientes de ce qui les attendait.

C'est ainsi que les trois auteurs ont cherché à recueillir la parole directe des jeunes femmes en question sur le calvaire qu'elles ont vécu. Ils en ont fait une pièce de théâtre, Le septième Kafana, qui fait l'objet de la présente étude.

Mais à la différence du simple théâtre documentaire, l'ambition des auteurs était surtout de participer, avec les moyens et la popularité de leur art, à la prévention de la traite des prostituées, en les avertissant de ce qu'elles risquaient, au moyen de témoignages de première main.

La prostitution est certes vieille comme le monde, en particulier dans cette région du monde, marche entre empires rivaux; mais il s'agit, dans le cas présent, d'un phénomène beaucoup plus féroce, celui de la prostitution forcée, avec des moyens de contraintes d'une violence extrême qui ont parfois conduit les jeunes prostituées à la mort.

Un précédent est rapporté par l'écrivain italien Curzio Malaparte qui, lorsqu'il était correspondant de guerre en Moldavie, pendant la Seconde Guerre mondiale, évoquait, dans le roman Kaputt, paru en 1946, au chapitre «Les jeunes filles de Soroca», des cas de même nature en Transnistrie, où la $11^{\mathrm{e}}$ armée allemande capturait des jeunes filles juives, les prostituait sous la menace d'être fusillées et les éliminait ensuite.

Dans le langage des femmes moldaves qui ont été envoyées, le plus souvent, dans les pays en guerre de l'ancienne Yougoslavie, ou en Albanie, kafana veut dire bordel. Le titre de la pièce vient de ce que le septième bordel dans lequel elles seraient amenées à travailler passait pour être le dernier, avant de perdre la raison ou la vie.

L'étymologie de ce terme, dans lequel les roumanophones reconnaîtront leur cafenea, est le turc kahvehane, débit de boisson, qui a pris en serbo-croate le sens de cabaret: lieu de plaisir, maison close. Le mot turc est lui-même composé du mot arabe qahouah, café, et du mot persan khaneh, maison. 
Faire du théâtre sur ce sujet délicat a été un pari osé, entre le théâtre militant, souvent perçu comme rébarbatif, et le risque de tomber dans un voyeurisme malsain. Nous verrons comment Le septième Kafana a évité ces pièges.

\section{Commentaire sur la pièce Le septième Kafana}

\section{Présentation}

Les jeunes filles, les jeunes femmes ne voulaient plus attendre de jours meilleurs mais aller au-devant de ceux-ci, pour aider leur famille, soigner un proche, assurer un avenir à leurs enfants, vivre mieux, tout simplement. Elles ne savaient pas qu'elles seraient vendues - l'une dix-huit fois! -, que leur passeport leur serait confisqué, que leur résistance serait brisée par les moyens les plus rudes: viols, coups, chantage et épuisement. Ces violences ont accompagné celles des guerres de la décennie 1990-2000, en BosnieHerzégovine et dans les autres pays issus de la Yougoslavie, en Albanie, et même jusqu'en Tchétchénie; en Italie, Belgique, Tchécoslovaquie, en France aussi parfois, pour les moins malchanceuses.

Certaines ne sont jamais revenues, d'autres sont rentrées dans un cercueil, racontent les six femmes dont le témoignage est recueilli et qui disent ce qu'elles ont vécu, ce qu'elles ont subi. Et aussi ce qu'elles ne peuvent dire, l'impossible réponse à la question lancinante du médecin qui les suivait: «Tu restes ici ou tu repars?» Repartir pour où ? Pour le bordel où elles meurent à petit feu, ou pour le pays qui les hante et les maudit? «Mon frère l'aurait su, il m'aurait enterrée vivante», dit l'une d'elle. «Ma mère a beaucoup vieilli. On ne s'est pas prises dans les bras, on ne s'est pas embrassées, on n'a pas pleuré», dit une autre.

\section{Intérêt}

L'intention pédagogique est manifeste dans le fait de vouloir prévenir, comme une prophylaxie, les jeunes femmes à qui on proposerait un travail bien payé au-delà des frontières, les avertir de la rude réalité vécue par celles qui témoignent. Étant donné l'ampleur des dégâts de la prostitution forcée en Moldavie, cette pédagogie répond à l'évidence à une nécessité.

Il fallait, pour les auteurs, détruire le rêve insensé et faux d'un ailleurs radieux, dénoncer l'exploitation d'un peuple désespéré dont les femmes ont affronté le pire.

Pour autant, l'option «théâtre» n'est pas qu'un moyen au service d'une cause sociale légitime. Le théâtre vient également solliciter la conscience du 
lecteur ou du spectateur en le mettant devant la manifestation dramatique, scénique, de l'émotion que les témoignages des filles contraintes à la prostitution provoquent; il est donc ici pleinement dans sa fonction. Les personnages ont la consistance de personnages réels, l'intensité dramatique cerne très bien la vérité vécue par les filles, leur drame, leur enfermement dans le Kafana autant qu'en elles-mêmes, la répétitivité de certaines situations sans avenir, sans issue, parfaitement résumée par la réplique: "Tu restes ici ou tu repars?" prononcée comme un refrain par le médecin gynécologue pendant la «visite» et à laquelle aucune fille n'arrive à répondre Le "septième Kafana» est, nous l'avons vu, le dernier établissement où travaillent les prostituées avant d'y laisser la vie ou de sombrer dans la folie.

\section{Écriture dramatique}

La pièce donc est tout d'abord écrite à partir de témoignages directs recueillis par les auteurs puis mis en forme pour la scène, afin de donner à entendre les paroles des intéressées, les victimes elles-mêmes. L'écriture dramatique suit ce choix de façon manifestement scrupuleuse, jusqu'à la pudeur oserait-on dire. Ce sont les filles qui parlent; elles n'ont pas besoin de recourir à des artifices sordides, ni de se réfugier dans le commentaire; la vérité leur suffit. Le commentaire, entre voyeurisme et désintérêt de fond, est le fait des autres, à l'instar de l'envoyée spéciale de CNN [et peut-être de quelques pièces de théâtre concurrentes ].

L'écriture est ainsi très «efficace»: $s$ 'il y a quelques mots crus, nécessaires à l'expression d'une vérité dépouillée, il n'y a aucun mot grossier, en ce sens qu'il n'y a aucun mot déplacé. Nous ne décelons pas non plus d'ego d'auteur dans l'écriture, ce qui nous semble très important (et là aussi suffisamment rare pour que l'on se fasse un devoir de le mentionner).

Honnêteté ou habileté suprême des auteurs, le lecteur, ou le spectateur, n'est conduit à aucune morale autre que celle de ses propres réactions. Rien de sordide ou de récupérateur ne lui est proposé, mais mise face à face devant la détresse humaine et liberté de compatir.

On sent à travers la succession des séquences combien la mise en scène peut tirer parti visuellement des situations. En ce sens, on pourrait parler d'une pièce de metteur en scène (c'est d'ailleurs le cas de Mihai Fusu, un des trois auteurs), qui écrit en sachant qu'il n'a pas besoin de tout faire dire par des mots, et qui sait ce que la mise en mouvement des acteurs en synergie avec les mots qu'ils prononcent, apporte à l'oralité. 


\section{Traduction}

La pièce a été jouée en France à plusieurs reprises, dès octobre 2002, en version originale roumaine. Une traduction en a été donnée, l'année suivante, et publiée aux Éditions L'Espace d'un instant (Paris, 2004). La traduction colle parfaitement au texte original. Le traducteur est d'ailleurs un des auteurs lui-même, Mihai Fusu, ce qui est au moins une garantie de fidélité. Ce dernier s'est adjoint une co-traductrice de langue maternelle française, Danny-Aude Rossel, et leur collaboration a donné un très bon résultat aussi bien pour rendre le sens du texte que son niveau de langue, cru et pudique à la fois. Ainsi rendue, la pièce passe très bien en français, sans vulgarité et ne sent aucunement la traduction.

\section{Contexte}

Très fortement marquée par la situation sociale qui prévaut en Moldavie anciennement soviétique, cette pièce a donc été jouée à l'Ouest, en France en particulier. Quoique les faits rapportés par la pièce se soient passés principalement en Bosnie-Herzégovine, l'opinion française s'est sentie concernée par ce fait de société et s'est émue du sort fait à ces filles si durement soumises.

Mais le voyeurisme, le sordide, la récupération moralisante voire hypocrite, n'ont pas toujours été absents du traitement de ce fait de société, dans la presse comme sur scène. C'est ce qui fait toute la différence avec $L e$ septième Kafana, totalement dépourvu d'artifice et de vulgarité.

\section{En guise de conclusion}

Bâtie à partir de témoignages, cette pièce n'en est pas moins une pièce de théâtre à part entière, qui «fonctionne» comme telle. Concilier l'exigence et l'efficacité dramatique d'une part et l'intention pédagogique d'autre part est un exercice qu'il est difficile de réussir; Le septième Kafana est un modèle d'adresse et d'équilibre dans le genre.

C'est aussi une pièce qui traite avec beaucoup de délicatesse et sans jugement de valeur un sujet délicat: performance rare, là aussi, que nous nous plaisons à souligner.

\section{Bibliographie}

Crudu, D., Esinencu, N., Fusu, M., 2003, Le septième Kafana, trad. de Danny-Aude Rossel, Éd. L'Espace d'un instant, Paris.

Burlacu, R., 2009, La vie qu'on a. L'histoire d'une fille de l'Est [témoignage à la première personne], L'Harmattan, Paris. 